\title{
Giant Magneto-impedance Effect in Composite Wires with Different Core Layer
}

\author{
R. L. Wang ${ }^{1,2}, \quad$ X. Li ${ }^{1}, \quad$ X. H. Kong ${ }^{2}$, Y. X. Guo ${ }^{2}$, J. Z. Ruan ${ }^{1}$, Z. J. Zhao ${ }^{1, *}$
}

(Received 22 April 2013; accepted 13 June 2013; published online 30 June 2013)

\begin{abstract}
Composite structure materials were potential sensing elements for magnetic sensors due to Giant magnetoimpedance (GMI) effect. Two kinds of composite wires with different magnetic/non-magnetic structures were fabricated by using electroless deposition methods and the magnetoimpedance properties were investigated. The maximum GMI ratio of $114 \%$ was acquired at $60 \mathrm{MHz}$ in the composite wires with a ferromagnetic core, whereas, $116 \%$ of maximum GMI ratio was found in the composite wires with a conductive core at low frequency of $600 \mathrm{kHz}$. These results exhibit that the GMI ratio reaches the maximum when magnetoresistance ratio $\Delta R / R$ and magnetoinductance ratio $\Delta X / X$ make the comparative contributions to the total magnetoimpedance (MI). The obvious GMI effect obtained in the composite wires with conductive core frequency may provide a candidate for applications in magnetic sensors, especially at low frequencies.
\end{abstract}

Keywords: Composite wires; Electroless deposition; Giant Magnetoimpedance effect; Resistivity

Citation: R. L. Wang, X. Li, X. H. Kong, Y. X. Guo, J. Z. Ruan and Z. J. Zhao, "Giant Magneto-impedance Effect in Composite Wires with Different Core Layer", Nano-Micro Lett. 5(2), 140-144 (2013). http://dx. doi.org/10.5101/nml.v5i2.p140-144

\section{Introduction}

The giant magnetoimpedance (GMI) effect was firstly observed in Co-based amorphous wires by Mohri et al in 1992 [1]. It has attracted much interest due to its stability and small size for potential applications in highly sensitive magnetic sensors. Many researchers have investigated GMI effect in different soft magnetic materials including homogeneous and composite materials [2-6]. It was found that GMI origins from classical electrodynamics, which relates to inductive effect, skin effect and ferromagnetic resonance. GMI effect is a preferable way to verify domain structure and saturation magnetostriction coefficient of magnetic materials as a research tool [7]. Simultaneously magnetometer based on GMI effect was developed in practical applications [8].

GMI effect can be improved by using suitable amor- phous or nanocrystalline soft magnetic materials with special geometric structures (microwire, ribbon and thin film) under optimized heat-treatment conditions [9-15]. For composite structures, such as composite wires and sandwiched films, their GMI ratios are much larger than those of homogeneous samples with the same dimensions and of similar ferromagnetic materials. The possible mechanism is enhancement of the skin effect due to electromagnetic interactions. If an insulator layer is added between two layers, composite wires with ferromagnetic core (CWFC) and composite wires with conductive core (CWCC) can be produced $[16,17]$. The GMI effect may be further enhanced if the thickness of the insulator is suitable for CWCC. The influence of conductive layer for CWFC is not too much. The mechanism is still unclear until now.

Among a variety of techniques for preparation of composite wires, such as electrodeposition [10] and

\footnotetext{
${ }^{1}$ Department of Physics, Engineering Research Center for Nanophotonics \& Advanced Instrument, Ministry of Education, East China Normal University, Shanghai 200062, China

${ }^{2}$ Experimental Teaching Center of Physics, Shanghai Ocean University, Shanghai 201306, China

*Corresponding author. E-mail: zjzhao@phy.ecnu.edu.cn
} 
magnetic sputtering [12,13], electroless deposition was believed to be the most suitable method due to its ability to provide uniform surface and cost-effectiveness of the fabrication process [14]. In this work, CWFC and CWCC are prepared using an electroless deposition method. Their GMI properties were comparatively studied.

\section{Experimental}

The detailed fabrication processes of two kinds of composition wires were reported in our previous work [16]. Firstly, the amorphous microwires of $\mathrm{Fe}_{73.0} \mathrm{Cu}_{1.0} \mathrm{Nb}_{1.5} \mathrm{~V}_{2.0} \mathrm{Si}_{13.5} \mathrm{~B}_{9.0}$ were prepared by a glass-coated melt-spinning method [19]. Then, the microwires were annealed at $570^{\circ} \mathrm{C}$ for $30 \mathrm{~min}$ in nitrogen atmosphere to improve their soft magnetic properties. Finally, a layer of copper was deposited onto the microwires by using an electroless deposition method, and the composite wires with ferromagnetic core were obtained.

The composite wire with conductive core, that is, with $\mathrm{Cu} /$ insulator/NiCoP structure, was also produced by using an electroless deposition. The $\mathrm{Cu}$ wires were carefully cleaned with distilled water and their surface was activated by $\mathrm{Pb} / \mathrm{Sn}$ particles before being mounted and placed into the electroless deposition bath. The bath composition contains $\mathrm{NiSO}_{4} \cdot 6 \mathrm{H}_{2} \mathrm{O}, \mathrm{CoSO}_{4} \cdot 7 \mathrm{H}_{2} \mathrm{O}$, $\mathrm{Na}_{2} \mathrm{C}_{4} \mathrm{H}_{4} \mathrm{O}_{6} \cdot 2 \mathrm{H}_{2} \mathrm{O}$ and $\mathrm{Na}_{3} \mathrm{C}_{6} \mathrm{H}_{5} \mathrm{O}_{7} \cdot 2 \mathrm{H}_{2} \mathrm{O}$. Operating temperature was controlled at $363 \mathrm{~K}$ using a water bath and $\mathrm{pH}$ value is maintained at $\sim 11$. The deposition products were annealed at $200^{\circ} \mathrm{C}$ to improve their soft magnetic properties.

The surface and the thickness of the plated layer of the samples were observed on scanning electron microscope (SEM, JSM-5610LV). The GMI effect was measured on an impedance analyzer (HP4294A). The constant alternating current $I_{r m s}=5 \mathrm{~mA}$ flows through the metallic nucleus and the frequency $f$ ranges from $40 \mathrm{~Hz}$ to110 MHz. The ratio was defined as,

$$
\begin{aligned}
& \frac{\Delta Z}{Z}(\%)=100 \times \frac{Z\left(H_{e x}\right)-Z\left(H_{\max }\right)}{Z\left(H_{\max }\right)}(\%) \\
& \frac{\Delta R}{R}(\%)=100 \times \frac{R\left(H_{e x}\right)-R\left(H_{\max }\right)}{R\left(H_{\max }\right)}(\%) \\
& \frac{\Delta X}{X}(\%)=100 \times \frac{X\left(H_{e x}\right)-X\left(H_{\max }\right)}{X\left(H_{\max }\right)} \%
\end{aligned}
$$

where $Z, R, X$ are impedance, the real and imaginary component of the impedance, respectively. $H_{e x}$ and $H_{\max }$ are an arbitrary and maximum intensity of external magnetic field, respectively. The DC external magnetic field was generated by a pair of Helmholtz coils.

\section{Results and discussion}

Figure 1 shows a typical SEM view of the two kinds of composite wires. It can be seen that the wires are composed of different layers and the plated surface is smooth. For CWFC wires as shown in Fig. 1(a), the diameter of ferromagnetic core is $7.0 \mu \mathrm{m}$. The thickness of coated glass layer and outer conductive layer is respectively $1.0 \mu \mathrm{m}$. For CWCC wires as shown in Fig. 1(b), the diameter of copper core is $90 \mu \mathrm{m}$. The thickness of coated Polyester ethylene is $5.0 \mu \mathrm{m}$ and outer ferromagnetic layer is $7.0 \mu \mathrm{m}$. Figure 1(c) and $1(d)$ show the cross-section schematic diagrams of two kinds of wires.

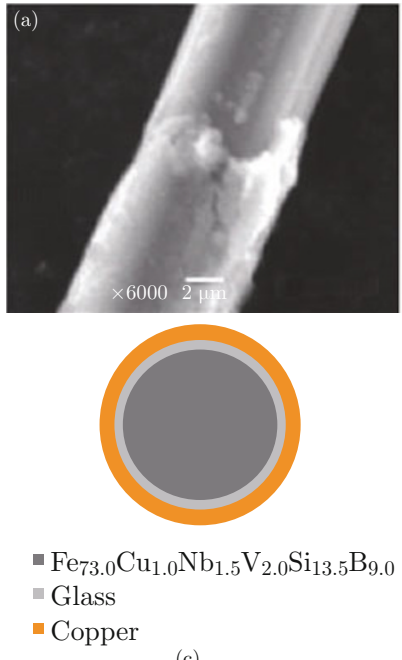

(c)
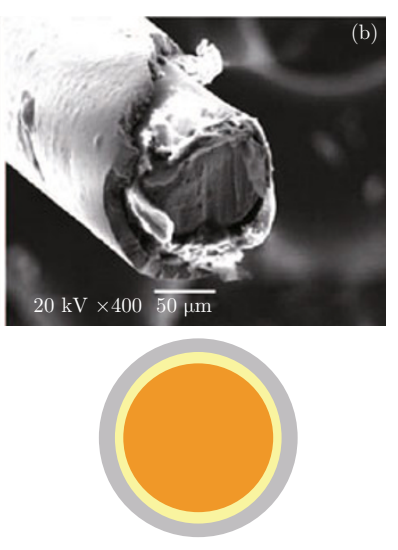

- Copper core

Polyester ethylene

$=\mathrm{NiCoP}$

(d)
Fig. 1 SEM images and sectional view of two kinds of composite wires. (a) and (c) for CWFC; (b) and (d) for CWCC.

The MI effect curves for glass-coated microwires annealed at $570^{\circ} \mathrm{C}$ tested at different frequencies are shown in Fig. 2. It can be seen that the maximum MI ratio is $118 \%$ at $60 \mathrm{MHz}$. Amonotonic decrease of the MI effect curve was observed at low testing frequency. However, at high testing frequency, the MI ratio increases initially with $H_{e x}$ till it reaches a peak and then falls with further increase of the external field. The MI effect is closely relative to the change in circumferential permeability of the wire with respect to the external field. At low frequencies, the domain wall displacement dominates to the magnetization process [18]. The circumferential permeability decreases with increasing external magnetic field. Along with the increasing of the driving frequency, the domain wall displacement is nearly damped and moment rotation will dominate the magnetization process.

Comparing to the microwires, the MI curves of the CWFC display the similar trend, as shown in Fig. 3. The maximum MI ratio is found to be $114 \%$ at $60 \mathrm{MHz}$. 


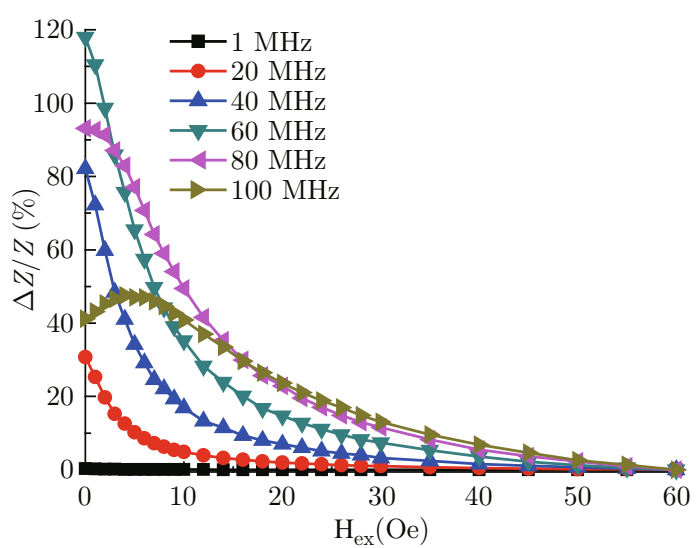

Fig. 2 MI ratio in variation with an external magnetic field for the microwires.

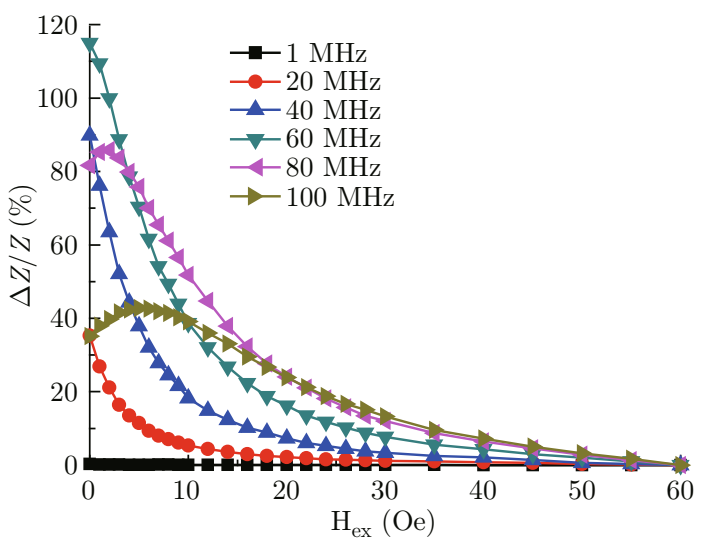

Fig. 3 MI ratio in variation with an external magnetic field for the CWFC.

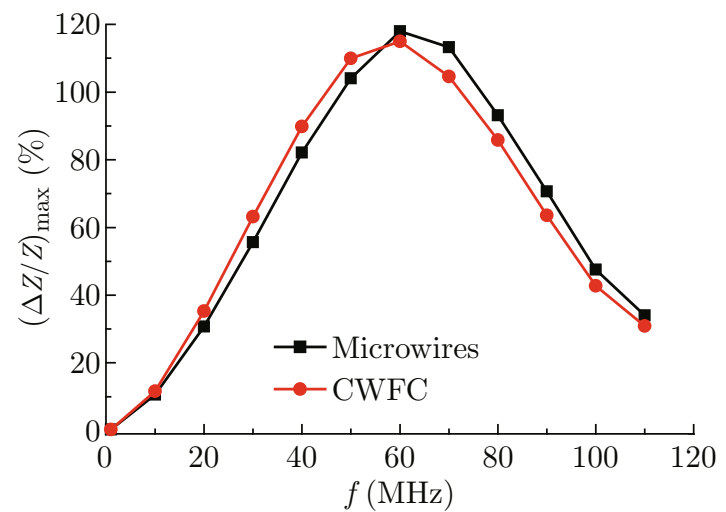

Fig. 4 The maximum GMI ratio with frequency for the CWFC and the microwires.

The dependence of the maximum $\mathrm{MI}$ ratio on the driving frequency for the CWFC and the microwires is given in Fig. 4. It can be seen that the GMI magnitude of the CWFC is different from that of the microwires. The MI ratio of the CWFC is higher than that of the microwires when the testing frequency is less than $60 \mathrm{MHz}$. However, the results are just the opposite when the testing frequency is more than $60 \mathrm{MHz}$. The circumferential permeability changes due to the electromagnetic interaction between the copper layer and ferromagnetic core [16].

The above mentioned results show that the eddy current in the copper layer is induced by the electromagnetic interactions, though $\mathrm{AC}$ current flows only through the ferromagnetic core. The magnetic field induced by the eddy current will in turn magnetize the ferromagnetic core, which will strengthen the dynamic magnetization at low frequencies. With the increase of the driving frequency, the influence of eddy current becomes more obvious. When the frequency is up to $60 \mathrm{MHz}$, the circumferential permeability is greatly reduced by eddy current loss. Thus the MI ratio decreases. However, the effect of copper layer is not remarkable in such kind of composite wires.

The external field dependence of MI ratio for the CWCC is illustrated in Fig. 5. It can be seen that the samples exhibit a peak MI ratio in variation with the external magnetic field, even at low testing frequencies. The MI ratio increases initially till it reaches a peak and then falls with further increase of the field. As it's mentioned above, the MI effect is relative to the changes in circumferential permeability with respect to the external field. The circumferential magnetization mainly proceeds via moment rotation for such kind of composite wire even at low frequencies. A maximum MI ratio of $116 \%$ was obtained at $600 \mathrm{kHz}$. The CWCC indicates obvious MI effect at low frequencies.

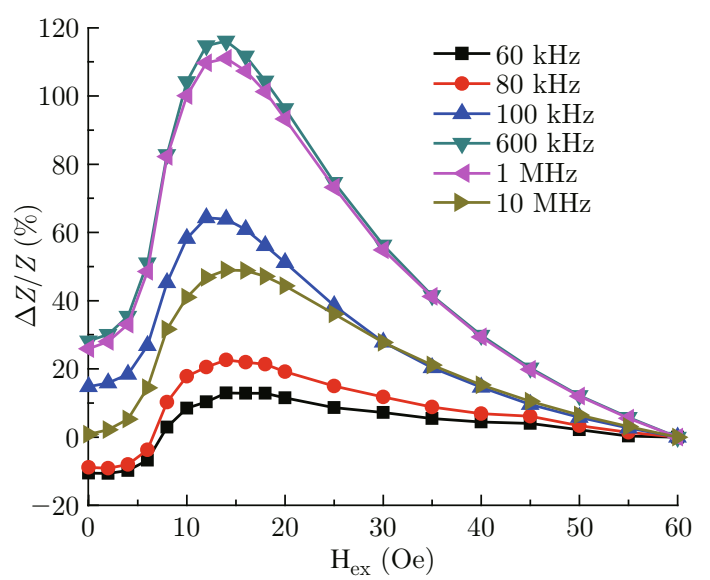

Fig. 5 MI ratio in variation with an external magnetic field for the CWCC.

To study the different influence of electromagnetic interaction on the two kinds of composite wires, the frequency dependences of $\Delta Z / Z, \Delta R / R, \Delta X / X$ of CWFC and CWCC have been depicted in Fig. 6 and its inset, respectively. It can be found that the maximum GMI ratio for the CWFC is $114 \%$ at $60 \mathrm{MHz}$, and its maximum $\Delta R / R$ ratio is $111 \%$ at $60 \mathrm{MHz}$. The CWCC reaches its maximum value of $116 \%$ at $600 \mathrm{kHz}$. Its maximum $\Delta R / R$ ratio achieves $205 \%$ at $2 \mathrm{MHz}$. 


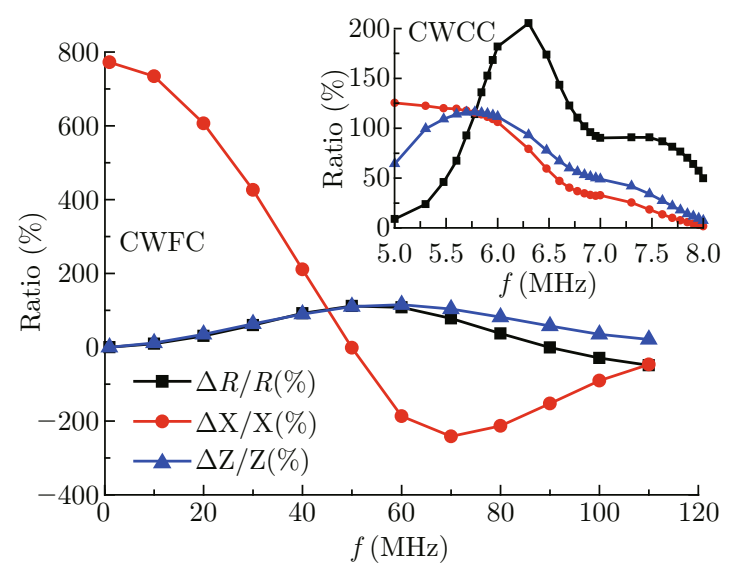

Fig. 6 Influence of frequency on $\Delta Z / Z, \Delta R / R, \Delta X / X$ of the CWFC, the inset shows frequency dependence of $\Delta Z / Z$, $\Delta R / R, \Delta X / X$ of the CWCC.

According to definition of impedance, $Z=R+i X$, the GMI ratio can be written as,

$$
\Delta Z / Z=\frac{\sqrt{(R+\Delta R)^{2}+(X+\Delta X)^{2}}-\sqrt{(R+X)^{2}}}{\sqrt{(R+X)^{2}}}
$$

Therefore, GMI ratio does not only depend on $\Delta R / R$ and $\Delta X / X$, but also on the ratio of $R$ to $X$. The resistivity of $\mathrm{Cu}$ core $\left(\sim 1.75 \times 10^{-8} \Omega \cdot \mathrm{m}\right)$ is very small, $R$ and $X$ of the CWCC is comparable at low frequencies. Both $\Delta R / R$ and $\Delta X / X$ make comparative contribution to total $\Delta Z / Z$ ratio. When the value of $\Delta R / R$ is equivalent to $\Delta X / X, \Delta Z / Z$ ratio reaches its maximum value. However, the resistivity of ferromagnetic core $\left(\sim 1.47 \times 10^{-6} \Omega \cdot \mathrm{m}\right)$ is much larger; two order magnitude higher than that of $\mathrm{Cu}$. The values of $R$ and $X$ for $\mathrm{CWFC}$ are not comparable at low frequencies until high frequencies. Though the value of $\Delta X / X$ is much higher than that of $\Delta R / R$ at low frequencies, $\Delta R / R$ makes main contribution to $\Delta Z / Z$. The different electrical and magnetic properties of the core lead to the different electromagnetic interaction. This is also the reason that there is no much influence of the copper layer on the microwires.

\section{Conclusions}

In summary, the difference of the MI effect in two kinds of composite wires was investigated. The maximum MI ratio of $114 \%$ was achieved in CWFC at 60 $\mathrm{MHz}$. Comparing to the microwires, the GMI effect of the CWFC strengths at low frequencies due to electromagnetic interaction. However, the effect is not remarkable. The CWCC shows better MI effect at lower frequencies, the maximum MI ratio of $116 \%$ was observed at $600 \mathrm{kHz}$. The results exhibit that the MI ratio reaches the maximum when both $\Delta R / R$ and $\Delta X / X$ make the comparative contribution to the total MI ratio. At low frequencies, $\Delta R / R$ has been obvious for
CWCC. Hence the CWCC has better MI properties at low frequencies.

\section{Acknowledgements}

The authors wish to thank Shanghai Automotive Science and Technology Development Foundation (SAISTDF/12-06), East China Normal University Program (78210142, 78210183), Shanghai Ocean University Program (A-2600-10-0054, B-5406-12-0012).

\section{References}

[1] K. Mohri, T. Kohhzawa, K. Kawashima, H. Yoshida and L. V. Panina, "Magneto inductive effect (MI effect) in amorphous wires", IEEE Trans. Magn. 28(5), 3150-3152 (1992). http://dx.doi.org/10.1109/20. 179741

[2] L. Kraus, Z. Frait, K. R. Pirota and H. Chiriac, "Giant magneto-impedance in glass-covered amorphous microwires", J. Magn. Magn. Mater. 254255, 399-403 (2003). http://dx.doi.org/10.1016/ S0304-8853(02) 00931-9

[3] A. Radkovskaya, A. A. Rakhmanov, N. Perov, P. Sheverdyaeva and A. S. Antonov, "The thermal and stress effect on GMI in amorphous wires", J. Magn. Magn. Mater. 249(1), 113-116 (2002). http://dx.doi. org/10.1016/S0304-8853(02)00516-4

[4] F. Alves, L. A. Rached, J. Moutoussamy and C. Coillot, "Trilayer GMI sensors based on fast stressannealing of FeSiBCuNb ribbons", Sensor Actuat APhys. 142(2), 459-463 (2008). http://dx.doi.org/10. 1016/j.sna.2007.06.004

[5] S. N. Kane, F. Alves, Z. Gercsi, F. Mazaleyrat, S. Gupte, H. Chiriac and M. Vazquez, "Study of magnetoimpedance effect in Co-Fe-Si-B glasscovered microwires", Sensor Actuat A-Phys. 129(1), 216-219 (2006). http://dx.doi.org/10.1016/j.sna. 2005.11 .044

[6] A. García-Arribas, J. M. Barandiarán and D. de Cos, "Finite element method calculations of GMI in thin films and sandwiched structures: size and edge effects", J. Magn. Magn. Mater. 320(14), e4-e7 (2008). http://dx.doi.org/10.1016/j.jmmm.2008.02.005

[7] D. X. Chen, J. L. Munoz, A. Hernando and M. Vazquez, "Magnetoimpedance of metallic ferromagnetic wires", Phys. Rev. B. 57(17), 10699-10704 (1998). http://dx.doi.org/10.1103/PhysRevB.57.10699

[8] B. Dufay, S. Saez, C. Dolabdjian, A. Yelon and D. Menard, "Development of a high sensitivity giant magneto-impedance magnetometer: comparison with a commercial flux-gate", IEEE Trans. Magn. 49(1), 8588 (2013). http://dx.doi.org/10.1109/TMAG. 2012. 2219579

[9] M. Ipatov, V. Zhukova, A. Zhukov and J. Gonzalez, "Magnetoimpedance sensitive to de bias current in amorphous microwires", Appl. Phys. Lett. 
97(25), 252507 (2010). http://dx.doi.org/10.1063/ 1.3529946

[10] A. Mishra, T. Sahoo, V. Srinivas and A. Thakur, "Investigation of magneto-impedance effect on electrodeposited $\mathrm{NiFe} / \mathrm{Cu}$ wire using inductance spectroscopy", Physica B. 406(3), 645-651 (2011). http://dx.doi. org/10.1016/j.physb.2010.11.070

[11] V. E. Makhotkin, B. P. Shurukhin, V. A. Lopatin, P. Y. Marchukov and Y. K. Levin, "Magnetic field sensors based on amorphous ribbons", Sensor Actuat A-Phys. 27(1), 759-762 (1991). http://dx.doi.org/10.1016/ 0924-4247 (91) 87083-F

[12] L. Chen, Y. Zhou, C. Lei, Z. M. Zhou and W. Ding, "Giant magnetoimpedance effect in sputtered single layered $\mathrm{NiFe}$ film and meander $\mathrm{NiFe} / \mathrm{Cu} / \mathrm{NiFe}$ film", J. Magn. Magn. Mater. 322(19), 2834-2839 (2010). http://dx.doi.org/10.1016/j.jmmm.2010.04.038

[13] L. Jiang, L. S. Tan, J. Z. Ruan, W. Z. Yuan, X. P. Li and Z. J. Zhao, "Intermittent deposition and interface formation on the microstructure and magnetic properties of $\mathrm{NiFe} / \mathrm{Cu}$ composite wires", Physica B. 403(18), 3054-3058 (2008). http://dx.doi.org/ 10.1016/j.physb. 2008.03.017

[14] N. Feldstein and J. A. Weiner, "Technique for controlling thio compound concentration in electroless plating baths", Anal Chem. 43(8), 1133-1134 (1971). http:// dx.doi.org/10.1021/ac60303a030
[15] J. Wang, L. Y. Zhang, P. Liu, T. M. Lan, J. Zhang, L. M. Wei, C. H. Jiang and Y. F. Zhang, "Preparation and growth mechanism of nickel nanowires under applied magnetic field", Nano-Micro Lett. 2(2), 134138 (2010). http://dx.doi.org:10.5101/nml.v2i2. p134-138

[16] R. L. Wang, Z. J. Zhao, L. P. Liu, W. Z. Yuan and X. L. Yang, "Giant magneto-impedance effect on nanocrystalline microwires with conductive layer deposit", J. Magn. Magn. Mater. 285(1-2), 55-59 (2005). http:// dx.doi.org/10.1016/j.jmmm. 2004.07.014

[17] L. Y. Shi, J. Z. Ruan, J. Zhang, Z. J. Zhao, H. B. Gao and U. Hartmann, "Enhancement of giant magneto-impedance effect in $\mathrm{Ni}_{80} \mathrm{Fe}_{20} / \mathrm{SiO}_{2} / \mathrm{Cu}$ composite wires", Physica B: Condensed Matter. 404(20), 3766-3770 (2009). http://dx.doi.org/10. $1016 / j \cdot$ physb. 2009.06.139

[18] R. Valenzuela, "Low-frequency magnetoimpedance: domain wall magnetization processes", Physica B: Condensed Matter. 299(3), 280-285 (2001). http:// dx.doi.org/10.1016/S0921-4526(01)00479-3

[19] K. Narita, N. Teshima, T. Agano and H. Funahashi, "Magnetic properties of 6.5-percent Si-Fe filament formed by a modified taylor", IEEE Trans. Magn. 16(3), 517-520 (1980). http://dx.doi.org/10.1109/ TMAG. 1980.1060640 